\title{
Energy efficiency of organized cluster election method in wireless sensor networks
}

\author{
Abderrahmane El Aalaoui, Abderrahmane Hajraoui \\ Department of Physics, Communication and detection systems laboratory, Faculty of Sciences, \\ University of Abdelmalek Essaadi Tetouan, Morocco
}

\section{Article Info \\ Article history: \\ Received Jun 25, 2019 \\ Revised Sep 26, 2019 \\ Accepted Oct 17, 2019}

\section{Keywords:}

Cluster head

Wireless sensor network

LEACH

Three dimensions

Energy efficiency

\begin{abstract}
In this paper, we propose a new approach to improve the protocol LEACH performance. It has been accomplished by increasing the energy balancing in clusters among all nodes to minimize the energy dissipation during network communications. The improved method is based on an optimized selection of cluster head. The development of this approach has indicated progress in terms of network lifetime, energy consumption and number of packets transferred to base station, compared to LEACH or other related extended spaces protocols. Mathematical analysis and simulations results show the effectiveness of the proposed approach. The energy consumption of WSN has been reduced up to about $3.5 \%$. The network life cycle is prolonged by $44 \%$. The number of packets sent has been increased by $185 \%$ than LEACH.
\end{abstract}

Copyright (C) 2020 Institute of Advanced Engineering and Science. All rights reserved.

\section{Corresponding Author:}

Abderrahmane El Aalaoui,

Faculty of Sciences, University of Abdelmalek Essaadi,

Mhannech II 93002 Tetouan, Morocco.

Email: elaalaoui.abderrahmane@gmail.com

\section{INTRODUCTION}

In Wireless Sensors Network (WSN), nodes are deployed in the real geographical environment with a random manner. WSNs have many analytical challenges. Each sensor is a small device in size, low cost and having low process capabilities. WSN's applications attracted great attention interest of researchers in recent years [1]. They have different applications such as monitor environmental aspects and physical phenomena like temperature measurement, audio and optical data exploitation, habitat monitoring, traffic control, patient healthcare monitoring, and underwater acoustic monitoring. Lately, the lifetime of each WSN is the most challenge of many researches. So far, many algorithms have been come up to solve the energy consumption of WSN. But due to their application specific nature, most of these algorithms are not suitable for wide threedimensional range of WSN. Also, random deployment of the sensors leads to increase the effect of energy consumption which decreases the lifetime of WSN.

A three-dimensional WSN is a set wireless sensor nodes distributed in a 3D space. Each sensor detects some events and sends their measurements toward a processing center called sink [2-3]. Node clustering is an effective technique for improving the energy efficiency and prolonging the network lifetime [4]. LEACH [4-5] is one of the first protocols which has used this technique and has been applied into the underwater environment by doing some changes [6-9].

At the cluster head, the principal treatments are done like reduction of redundant data and applying aggregation techniques that minimizing the data size and forward it to the BS [10]. Recently, many clustering techniques have been proposed for WSNs. In addition, many studies have been done to analyze existing clustering techniques and algorithms.

In the reference [11], its authors propose a three dimensional coverage model and an optimum deployment scheme for wireless sensor networks. In paper [12], they have presented a new approach for 
organizing the sensor network into several levels which use two techniques, a static one and a dynamic other. Authors in [13], have compared the energy consumption between the three-dimensional and the twodimensional space. In [14], authors have studied the protocol LEACH, applied in a homogeneous WSN with two-level of nodes density in order to reduce the energy consumption. In the paper [15], they have examined the LEACH-level, which considers the homogeneous WSN, in extended spaces and with multilevel of nodes density. Therefore, it can produce better performance. In [16], they have discussed the MLHEED, a multilevel heterogeneous network model applied in HEED protocol. In the paper [17], they discuss the HEED-Multilevel. That is enrichment over the HEED protocol, by analyzing the energy efficiency of splitting the network into levels with different densities of nodes. That is significantly reduces the system cost.

There are other versions of LEACH protocols such as follows: NR-LEACH [18] overcomes the random process selection, which leads to unexpected fail for some cluster heads in other LEACH versions, and it gives a good performance in the network lifetime and energy consumption comparing with previous version of LEACH protocols. LEACH-C, Centralized [19-20] in which each node location is a vital information for BS to run a centralized clustering algorithm to organize the clusters for current round. Most suitable nodes will be selected to represent a $\mathrm{CH}$, and the non- $\mathrm{CH}$ nodes will be distributed as cluster member nodes. The first dead node metric enhancement occurred in LEACH-C compared with traditional LEACH. LEACH-GA [21] protocol is a genetic algorithm grouping system. Every node sends its node identifier, area data, and $\mathrm{CH}$ choice in view of ideal rate of $\mathrm{CH}$ to the $\mathrm{BS}$. The $\mathrm{BS}$ applies genetic calculation operations on got data to discover the ideal limit likelihood and communicate it for group arrangement. Depending on Vice-LEACH [22-23] protocol, data aggregated by the CHs will constantly arrived the BS. Thusly, there is no convincing motivation to select another $\mathrm{CH}$ each time. The network lifetime extended, but an overhead based on $\mathrm{CH}$ selection arises. Multi-hop LEACH [24] protocol recommended to handle the power consumption load. All nodes are immobile, and BS is fixed. The process of $\mathrm{CH}$ selection is based on random selection. It gets advantage of multihop technique to minimize the total power consumption. Critical issue is denoted when one or more intermediate $\mathrm{CHs}$ died or failed.

In this paper, a new mechanism of cluster head selection has been proposed in LEACH protocol for wide real sensor network area. We suggest a new algorithm named organized LEACH (O-LEACH) which localizes the clusters that have the shortest distance from the Base Station. Simulation results validate the performance of the proposed approach.

\section{RESEARCH METHOD}

Authors in [12] have defined the energy consumption of three dimensional WSN as (1). Figure 1, shows the energy consumed in WSN with different numbers of cluster head that have same distance far from the base station.

$$
E_{\text {Total }}=l\left(2 N E_{\text {elec }}+N E_{D A}+c \epsilon_{m p} d_{\text {toBS }}^{4}+N \varepsilon_{f S} \frac{3}{10}\left(\frac{3}{4 \pi c}\right)^{\frac{2}{3}} M^{2}\right)
$$

With:

$E_{\text {Total }}$ : Energy consumed by WSN per round

$d_{t o B S}: \quad$ Distance of cluster form base station

$c: \quad$ Number of clusters

$N$ : Number of nodes

$l$ : $\quad$ Number of message bits with respect to $d_{t o B s}$ :$$
\frac{\partial E_{T o t a l}}{\partial d_{t o B S}}=0
$$$$
4 l c \epsilon_{m p} d_{t o B S}^{3}=0
$$

With : $x=d_{t o B S}$, we find the function as follow :

$$
f(x)=4 l c \epsilon \operatorname{mpx} 3
$$




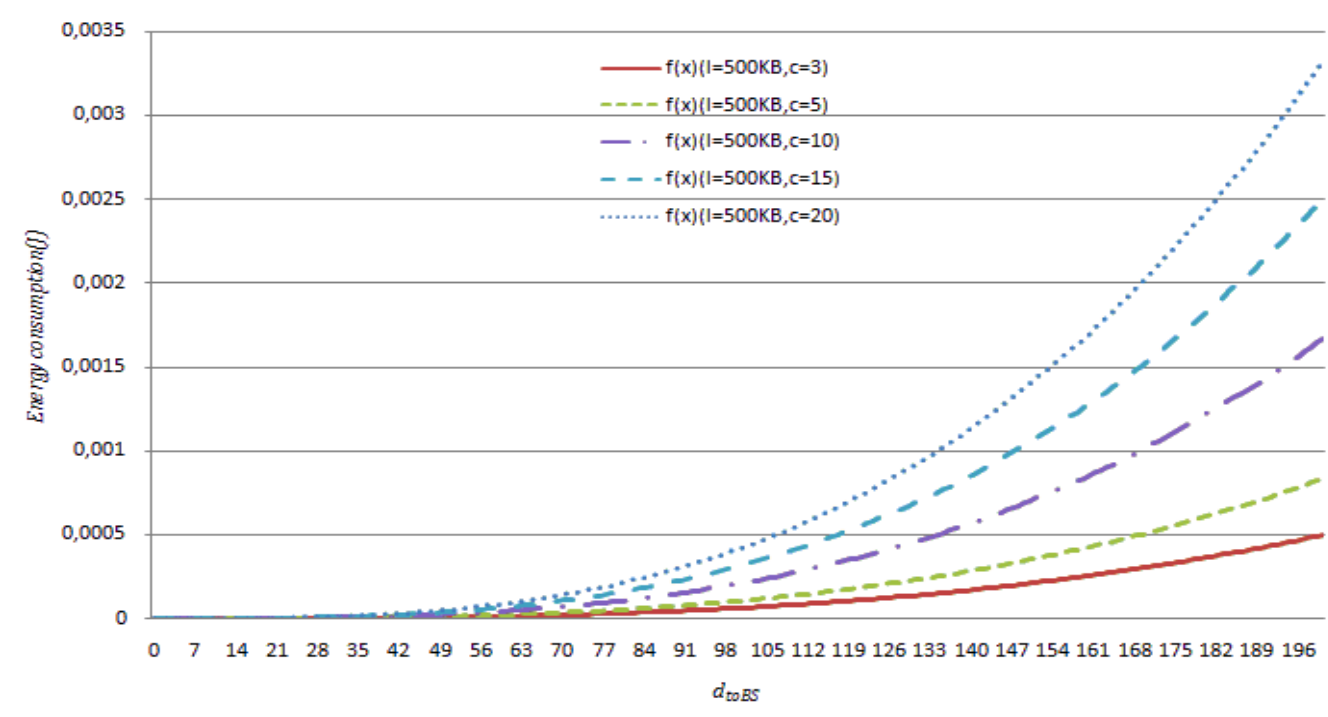

Figure 1. Variation of energy consumption for different values of distance to base station

All graphs indicate the same direction when the distance from any cluster head to base station is the minimum. In 50 meter, the energy increases for each graph and more when the distance increases. When the distance increases we assume that the WSN that have minimum number of clusters consumes less of energy than that have maximum number of clusters.

Our proposition is to find the nearest nodes of base station and select them as clusters as the figure below shows. This selection permits efficient energy consumption especially in wide distances as the Figure 1 told us. Selection of nodes nearest of base station as clusters as shown in Figure 2.

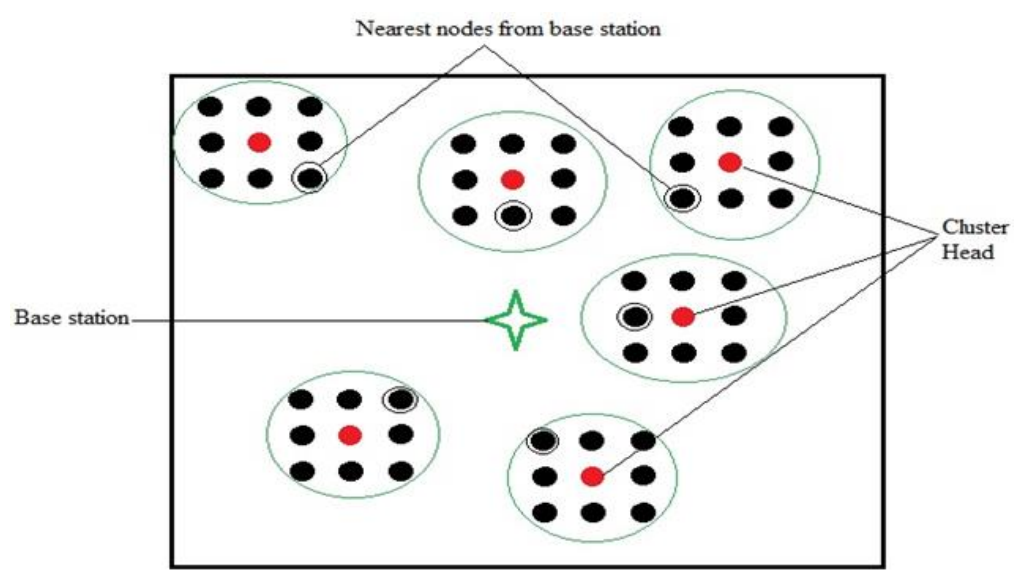

Figure 2. Selection of nodes nearest of base station as clusters

\subsection{Organized LEACH}

LEACH is a cluster-based protocol. It includes a communication of distributed clusters. LEACH randomly selects a few nodes as cluster-heads and rotates this role to evenly distribute the energy load among the sensors in the network. In LEACH, each cluster-head compresses data arriving from nodes and sends an aggregated packet to the BS [25].

LEACH protocol is divided into rounds. Each round consists of two phases: Set-up Phase and Steady Phase. The set-up phase is further divided into 2 parts. LEACH forms clusters by using a distributed algorithm where nodes make autonomous decisions without any centralized control. As shown in Figure 3, in the advertisement phase, $\mathrm{CHs}$ inform their neighborhood that they become $\mathrm{CHs}$. The non-CH nodes pick the advertisement packet with the strongest received signal. Each node decides independent of other nodes if 
it will become a $\mathrm{CH}$ or not. This decision is made by looking into account when the node served as a $\mathrm{CH}$ for the last time (means the node that hasn't been a $\mathrm{CH}$ for long time is more likely to elect itself than nodes that have been a $\mathrm{CH}$ recently). This is done according to a distance threshold. This threshold value depends upon the desired percentage $\mathrm{p}$ to become a cluster head, the current round $\mathrm{r}$, and the set of nodes that have not become cluster heads in the last $1 / p$ rounds. It is denoted by $\mathrm{G}$. Based on all messages received within the cluster, the $\mathrm{CH}$ creates a TDMA schedule, pick a CSMA code randomly, and broadcast the TDMA table to cluster members. Every node wanting to be the cluster head chooses a value, between 0 and 1. If this random number is less than the threshold, then the node becomes the cluster head for the current round. Then each elected $\mathrm{CH}$ broadcasts an advertisement message to the others nodes in the network to invite them to join their clusters. Based upon the strength of the advertisement signal, the non-cluster head nodes decide to join the clusters.In the set-up phase, the cluster head nodes are randomly selected from all sensors and several clusters are constructed dynamically.

Our O-LEACH analyzes each $\mathrm{CH}$ and its joined nodes then search which of them is the nearest one of the Base Station. If the chosen $\mathrm{CH}$ is the nearest one, it remains unchanged. If another node has the minimum distance of the base station, it will be the new cluster head and broadcasts an advertisement message to the ancient cluster head, to change it status to normal node and an advertisement message to the rest of the group nodes invites them to join the new cluster head. Following flowchart shows the procedure in the set-up phase:

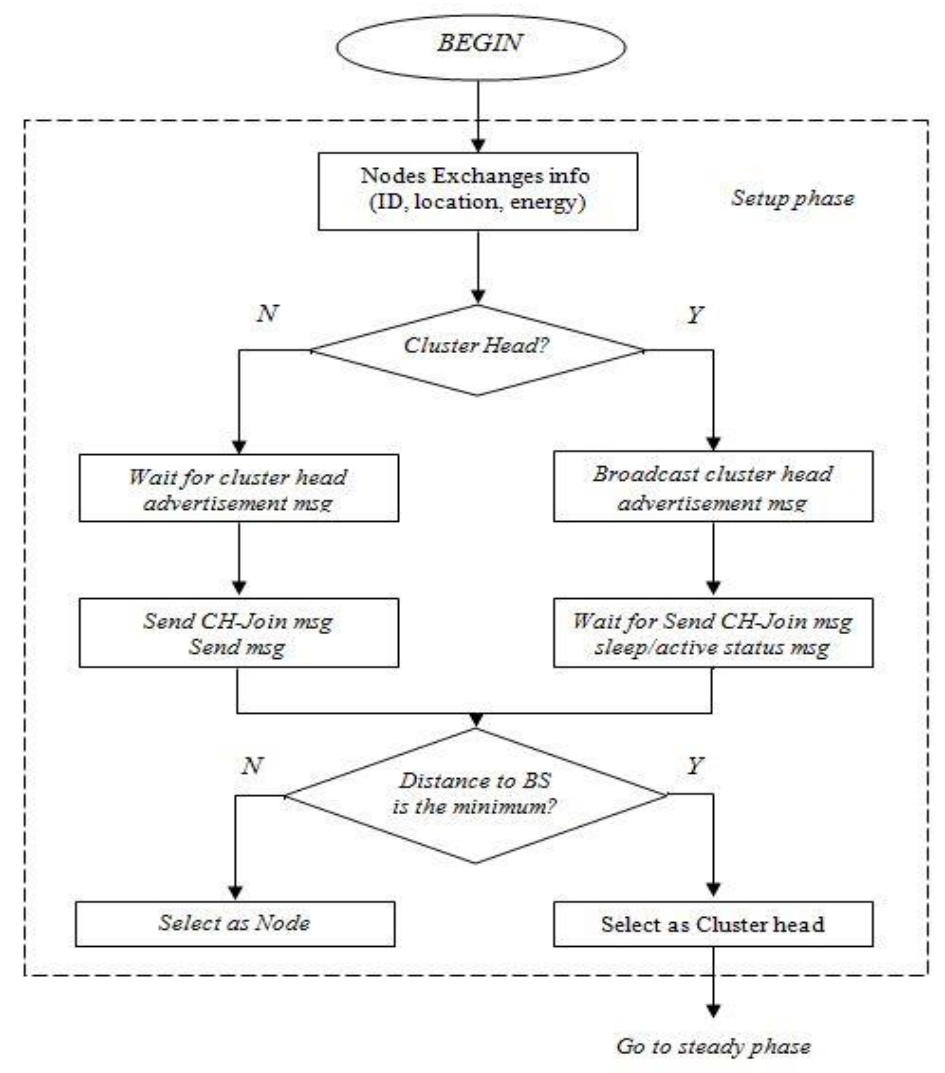

Figure 3. Flowchart of the O-LEACH

\subsection{Network Model}

a. $\quad \mathrm{N}$ sensors are uniformly distributed in a space 3D. It is a rectangular field $\mathrm{S}=\mathrm{D} \times \mathrm{D} \times \mathrm{D}$. The base station is positioned in the center of the space region.

b. All nodes are deployed randomly.

c. Each sensor can sense the environment in a sphere field of a radius $r$.

d. All sensors are homogeneous. They have the same capacities.

e. All nodes have a particular identifier (ID) allocated to them. Each cluster head coordinates the MAC and routing of packets within its cluster. 


\section{RESULTS AND DISCUSSION}

The proposed approach is simulated using MATLAB, 3D Elliptical Gaussian distribution is implemented. The WSN is represented by 100 nodes that are deployed in different regions of a cubical field. The base station is located at cube's center. The chosen initial energy is $5 \times 10-3 \mathrm{~J}$ for each sensor. The simulation of the proposed approach is repeated at an average of 20 times. The simulation results are compared with the simulation results of LEACH protocol. This comparison is based on four performance metrics. It includes network lifetime, number of packets received at the base station, Average distance of cluster heads from the base station and total energy dissipation. The proposed simulation parameters are shown in Table 1.

Table 1. Simulation Parameters

\begin{tabular}{cc}
\hline Parameter & Value \\
\hline $\mathrm{N}$ & 100 \\
$E_{\text {elec }}$ & $50 \mathrm{~nJ} / \mathrm{bit}$ \\
$E_{D A}$ & $5 \mathrm{pJ} / \mathrm{bit}$ \\
$E_{f s}$ & $10 \mathrm{pJ} / \mathrm{bit} / \mathrm{m}^{2}$ \\
$E_{\text {amp }}$ & $0.0013 \mathrm{pJ} / \mathrm{bit} / \mathrm{m}^{2}$ \\
$l$ & $500 \mathrm{bytes}$ \\
$p$ & 0.1 \\
Rounds & 100 \\
\hline
\end{tabular}

\subsection{Network lifetime}

Network lifetime is defined as to be the maximum amount of time between First Node Death (FND) and Last Node Death (LND). Longer stability period for the network is an important requirement, because a data loss in any node affects the final results. Figure 4 shows the lifetime for LEACH and the proposed one of the O-LEACH. It is observed that in proposed LEACH, the FND is in round 7 but it is in round 3 for the classical LEACH. In round 23, the normal LEACH lost the last sensor compared with the result of proposed LEACH. It keeps 2 nodes after this event. Also, there is an improvement in the stability period, which is required for some applications.

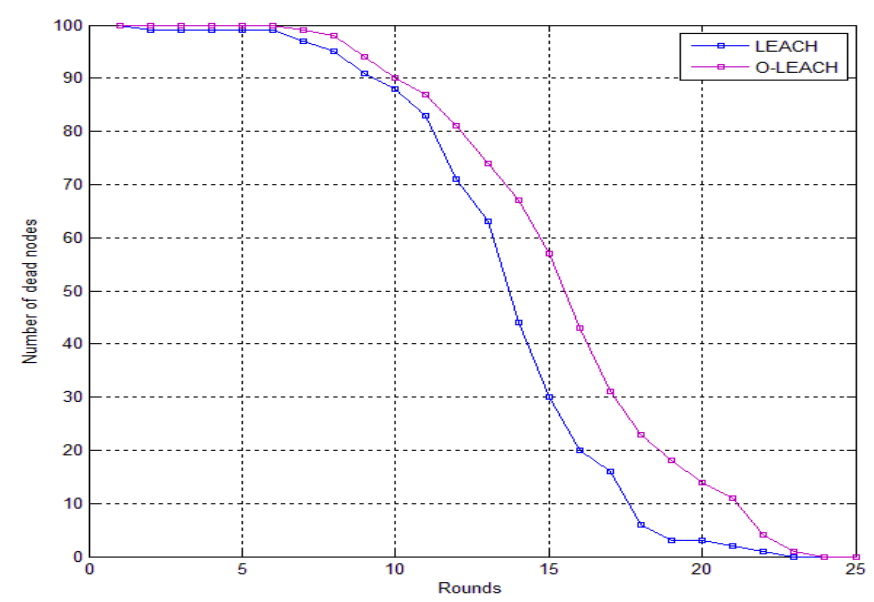

Figure 4. Number of dead nodes versus Rounds in $S=100 \mathrm{~m} 3$

\subsection{Number of Packets Sent to BS}

In the proposed approach, the number of packets received at the base station is more than the numbers of packets received by LEACH as shown in Figure 5. The number of packets is estimated by energy consumption. More is the stability in energy consumption more is the number of packets received at the base station. This enhancement is obtained due to cluster head selection method. It ensures a balanced generation of cluster heads. This balance causes stability in the indicated selection. It leads to regular in energy dissipation. 


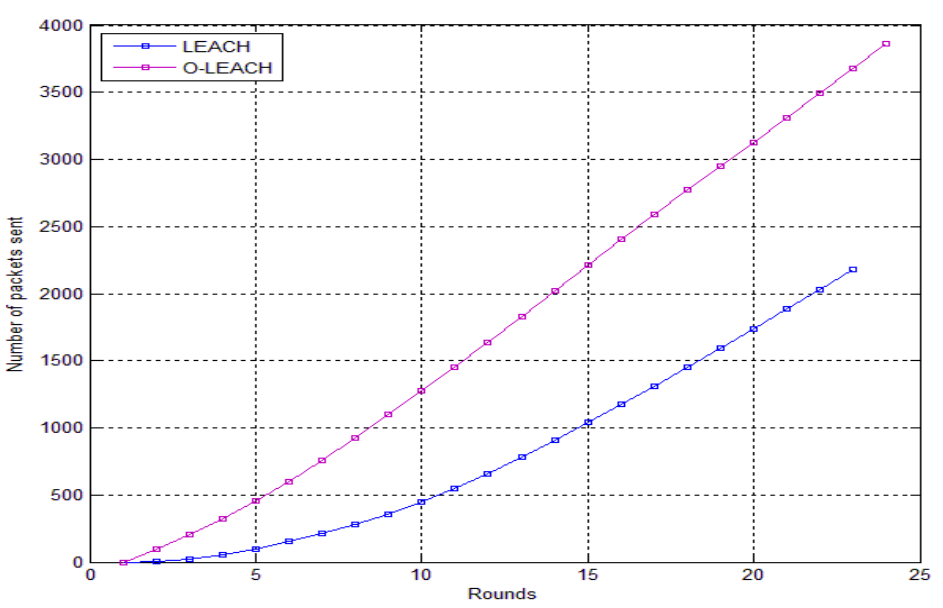

Figure 5. Number of packets versus Rounds in $S=100 \mathrm{~m} 3$

\subsection{Average Distance of $\mathrm{CH}$ from $\mathrm{BS}$}

Figure 6 shows the average distance of cluster head from the base station. It is extremely impacting on the energy balanced of WSN. As the average distance of each cluster head increased, the energies of the nodes near or far from center are consumed by balanced manner due to the distribution of cluster in the whole space of WSN. On the other hand, as the average distance of cluster head nodes is minimized, the energies of only the nodes far from center are severely consumed. It is due to the distribution of cluster in the space near from BS. Therefore, these nodes will dead earlier.

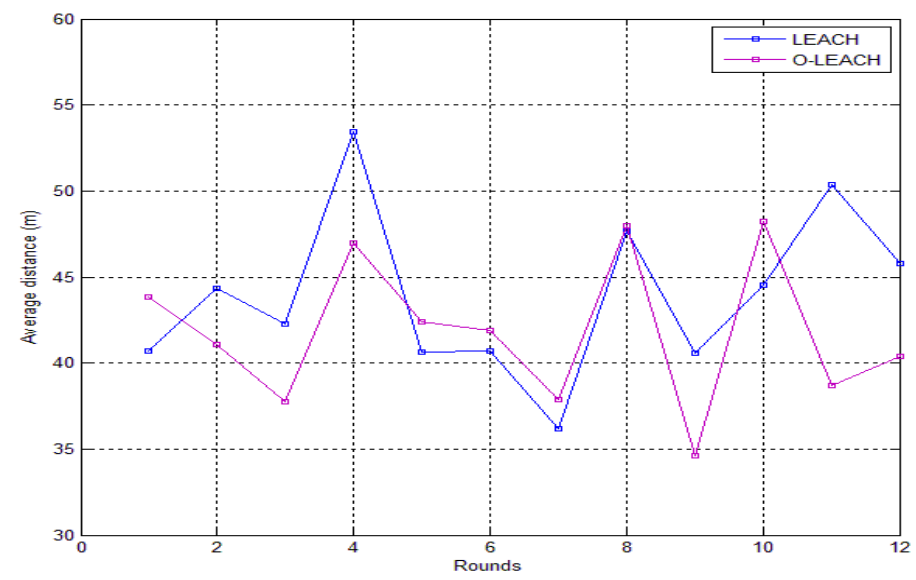

Figure 6. Average distance versus Rounds in $S=100 \mathrm{~m} 3$

\subsection{Energy consumption}

Energy consumption means the total energy consumed by the network to perform transmission, reception and data aggregation. A comparison is performed among different approaches based on the energy consumption in both cluster heads and cluster members. For both of them, the simulation is run on the number of nodes equal to 100, 200, 300, 400 and 500. As shown in Figure 7, the O-LEACH approach achieves the minimum energy consumption compared with all other approaches. In addition, the enhancements of energy consumption are satisfied for both cluster heads and cluster members. This improvement is achieved due to the switch off and sleep mode assigned to each sensor after transmission and proper $\mathrm{CH}$ selection that mentioned before. Finally, the Switch off and sleep mode protect the sensor from the inefficient transmission and cluster head from an idle listening stage. Table 2, shows energy consumption and alive nodes number for both LEACH and O-LEACH for different sensor numbers after ten rounds. 
Table 2. Alive Nodes Number and Energy Consumption of WSN after Ten Rounds in in S=100 M3

\begin{tabular}{|c|c|c|c|c|c|}
\hline Nodes number & Protocol type & Energy consumption $(\mathrm{J})$ & Rate Energy & Alive nodes number & Rate Alive Nodes \\
\hline \multirow[t]{2}{*}{$\mathrm{N}=100$} & LEACH & 0.4431 & - & 82 & - \\
\hline & O-LEACH & 0.4296 & $-3.14 \%$ & 88 & $7.32 \%$ \\
\hline $\mathrm{N}=200$ & LEACH & 0.8555 & - & 166 & - \\
\hline \multirow[t]{2}{*}{$\mathrm{N}=300$} & LEACH & 1.2770 & - & 256 & - \\
\hline & O-LEACH & 1.2770 & $0 \%$ & 279 & $9 \%$ \\
\hline $\mathrm{N}=400$ & LEACH & 1.6826 & - & 337 & - \\
\hline $\mathrm{N}=500$ & O-LEACH & 2.0487 & $-2.82 \%$ & 473 & $9.24 \%$ \\
\hline
\end{tabular}

With:

Rate Energy $=\frac{\text { Energy consumption }(O-L E A C H)-\text { Energy consumption }(L E A C H)}{\text { Energy consumption }(\text { LEACH })}$

Rate Alive Nodes $=\frac{\text { Alive nodes number }(O-L E A C H)-\text { Alive nodes number }(L E A C H)}{\text { Alive nodes number }(L E A C H)}$

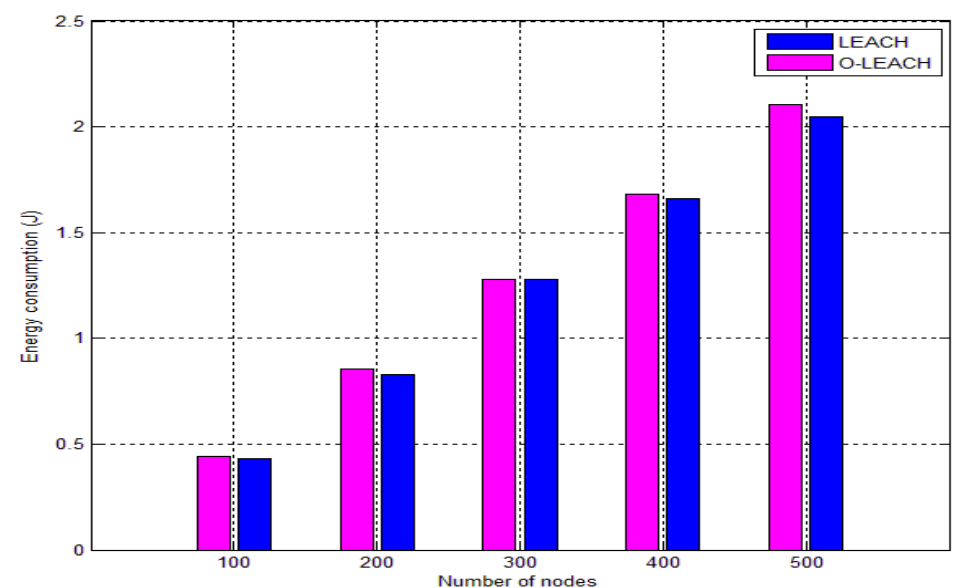

Figure 7. Energy consumption versus number of nodes

The following tables show the impact of our proposed protocol on different other protocol LEACH in wide distance with levels or without:

Table 3 shows the improvement of the WSN network lifetime and number of packets sent in different spaces $(S=100 \mathrm{~m} 3$ and $S=200 \mathrm{~m} 3)$.

Table 3. Number of Dead Nodes and Packets Sent Different Dimensions D

\begin{tabular}{cccccccc}
\hline Dimension & Protocol type & FND & Rate FND & LND & Rate LND & Packets Number & Rate Packets \\
\hline \multirow{2}{*}{$100 \mathrm{~m}$} & LEACH & 2 & - & 23 & - & 2176 & - \\
& O-LEACH & 7 & $250 \%$ & 24 & $4.35 \%$ & 3859 & $77.34 \%$ \\
& LEACH & 1 & - & 16 & - & 1185 & - \\
& O-LEACH & 4 & $300 \%$ & 23 & $43.75 \%$ & 3366 & $184 \%$ \\
\hline
\end{tabular}

Rate FND $=\frac{F N D(O-L E A C H)-F N D(L E A C H)}{F N D(L E A C H)}$

Rate $L N D=\frac{L N D(O-L E A C H)-L N D(L E A C H)}{L N D(L E A C H)}$

Rate Packets $=\frac{\text { Packets Number }(O-L E A C H)-\text { Packets Number }(\text { LEACH })}{\text { Packets Number }(L E A C H)}$ 
Table 4 presents the performance comparison of the proposed approach with 3D LEACH-nlevel protocols [14-15], in different spaces $(S=200 \mathrm{~m} 3$ and $S=300 \mathrm{~m} 3)$.

Table 4. Number of Dead Nodes and Packets Sent in Different Dimensions D with Levels

\begin{tabular}{cccccccc}
\hline Dimension & Protocol & FND & Rate FND & LND & Rate LND & Packets Number & Rate Packets \\
\hline \multirow{2}{*}{$200 \mathrm{~m}$} & LEACH Two-Level & 2 & - & 19 & - & 1407 & - \\
& O-LEACH Two-Level & 2 & $0 \%$ & 22 & $15.79 \%$ & 3222 & $129 \%$ \\
\multirow{2}{*}{$300 \mathrm{~m}$} & LEACH Three-Level & 1 & - & 16 & - & 912 & - \\
& O-LEACH Three-Level & 1 & $0 \%$ & 17 & $6.25 \%$ & 1864 & $104 \%$ \\
\hline
\end{tabular}

\section{CONCLUSION}

In this paper, an efficient approach to enhance the routing procedures in the LEACH protocol for three-dimensional WSNs has proposed. The proposed method aims to follow the random selection of cluster head and to divide the WSN to groups. Each group has a cluster head and nodes member. By fixing the cluster head choice into the nearest node of each group from base station, the proposed method has enhanced the energy consumption of the wireless sensor nodes. Therefore, the lifetime of the WSN has extended compared with the classical LEACH protocol. The implementation results of the proposed approach justify the introduced improvement. A comparison of the proposed approach with classical LEACH and other multilevels $\mathrm{LEACH}$, in terms of network lifetime, of energy consumption and of packets transferred to BS, has had yields better outcomes. In a future work, we will attempt to apply the proposed approach to the Fuzzy LEACH protocol.

\section{ACKNOWLEDGEMENTS}

The authors would like to thank, for their technical support, Ph.Ds. Saad Chakkor, El Ahmadi Cheikh, Mostafa Baghouri and Abdelkader Bourzek from Abdelmalek Essaadi University Morocco. A great appreciation is also extended to colleagues: Pr Eric Busvelle from Dijon University France and also Pr Jean Louis Lilien from Liege University Belgium, for their continued help. The authors would also like to add a special thanks to Pr A. El Khamlichi from AbdelmalekEssaadi University Morocco, for his good assistance.

\section{REFERENCES}

[1] I. F. Akyildiz, W. Su, Y. Sankarasubramaniam, and E. Cayirci, "Wireless sensor networks: a survey". Computer networks, vol. 38, no. 4, pp. 393-422, 2002.

[2] H.M. Ammari, S.K. Das, "Critical Density for coverage and connectivity in three dimensional wireless sensor networks using percolation theory". IEEE Trans. Parallel Distrib, vol. 20, Issue.6, pp. 872-885. June 2009.

[3] Kay Romer and Friedemann Mattern. "The Design Space of Wireless Sensor Networks". IEEE Wireless Communications, Vol. 11, Iss. 6, pp. 54-61.Dec. 2004.

[4] Wendi R. Heinzelman, AnanthaChandrakasan, and HariBalakrishnan, "Energy efficient communication protocol for wireless micro sensor networks", 33rd Annual Hawaii International Conference on System Sciences, pp. 1-10, 2000.

[5] W. Heinzelman, A. Chandrakasan, and H. Balakrishnan, "An application specific protocol architecture for wireless micro sensor networks", IEEE Transactions on Wireless Communications, vol. 1, Iss. 4, pp. 660-670, October 2002.

[6] Xia Li, Yongqian Wang, and Jingjin Zhou, "An energy-efficient clustering algorithm for underwater acoustic sensor networks", International Conference on Control Engineering and Communication Technology (ICCECT), pp. 711-714, Dec. 2012.

[7] X.Li, S.-L.Fang, and Y.C. Zhang, "The study on clustering algorithm of the underwater acoustic sensor networks,"14th International Conference on Mechatronics and Machine Vision in Practice (M2VIP), pp. 4-6, December 2007.

[8] Guangsong Yang, Mingbo Xiao, En Cheng, and Jing Zhang, "A cluster-head selection scheme for underwater acoustic sensor networks", International Conference on Communications and Mobile Computing (CMC), vol. 3, pp. 188-191, April 2010.

[9] Liu, G., and Wei, C., "A new multi-path routing protocol based on cluster for underwater acoustic sensor networks", International Conference on Multimedia Technology (ICMT), pp. 91-94, 2011.

[10] M. Elshrkawey, S. Elsherif, M. ElsayedWahed, "An Enhancement Approach for Reducing the Energy Consumption in Wireless Sensor Networks", Journal of King Saud University-Computer and Information Sciences, Vol. 30, Iss. 2, pp. 259-267, 2018.

[11] Fu Xiao, Yang Yang, Ruchuan Wang and Lijuan Sun. "A Novel Deployment Scheme Based on Three-Dimensional Coverage Model for Wireless Sensor Networks". The Scientific World Journal, Volume 2014, Article ID 846784, 7 pages, 2014. 
[12] E. Alnawafa and I. Marghescu."New Energy Efficient Multi-Hop Routing Techniques for Wireless Sensor Networks: Static and Dynamic Techniques", Sensors 2018, 18, 1863; doi: 10.3390/s18061863.

[13] M.Baghouri, A.Hajraoui, S.Chakkor. "Three Dimensional Low Energy Adaptive Clustering Hierarchy for Wireless Sensor Network". $19^{\text {th }}$ International Conference on Communications (part of CSCC'15), Zakynthos Island, Greece, pp. 214-218, July 2015.

[14] A. El Aalaoui and A. Hajraoui, "Multi-Level Deployment in Extended Three-Dimensional Spaces for WSN", 6th International Conference on Automatic \& Signal Processing (ATS-2018),Proceedings of Engineering and Technology-PET, Vol. 35, pp. 32-39, 2018.

[15] A. El Aalaoui and A. Hajraoui, "Remarkable Performance of Multilevel Deployment in Wireless Sensor Networks", International Journal of Computer Science, Communication and Information Technology (CSCIT). Vol. 6, pp. 9-18, 2018.

[16] Samayveer Singh, "Energy efficient multilevel network model for heterogeneous WSNs", Engineering Science and Technology, an International Journal, Vol. 20, No. 1, pp. 105-115, 2017.

[17] A. El Aalaoui and A. Hajraoui, "Analysis of energy efficiency for the extended WSN using a multilevel HEED protocol", International Journal of Advanced Research in Science, Engineering and Technology (IJARSET). Vol. 6, Issue 3, pp. 8541-8551, March 2019.

[18] A. Al-Baz and A. El-Sayed, "A new Algorithm for Cluster Head Selection in LEACH protocol for Wireless Sensor Networks", International Journal of Communication Systems, Engineering and Technology (IJARSET). Vol. 31, Issue 1, pp. 1-13, January 2018.

[19] Wu XH, Wang S. "Performance comparison of LEACH and LEACH-C protocols by NS2". In: Proc. 9th International Symposium on Distributed Computing and Applications to Business, Engineering and Science. pp. 254-258, 2010.

[20] Gnanambigai J, Rengarajan N, Anbukkarasi K. "Leach and its descendant protocols: a survey". International Journal of Communication and Computer Technologies. Vol. 1, No. 3, Issue 2, pp. 15-21, September 2012.

[21] Liu J-L, Ravishankar CV. "LEACH-GA: genetic algorithm-based energy-efficient adaptive clustering protocol for wireless sensor networks". International Journal of Machine Learning and Computing. Vol. 1, No 1, pp. 79-85, 2011.

[22] Sindhwani N, Vaid R. "V-Leach: an energy efficient communication protocol for WSN". Journal Mechanica Confab, Vol. 2, No 2. pp. 79-84, Feb-March 2013.

[23] Ahlawat A, Malik V. "An extended vice-cluster selection approach to improve V Leach protocol in WSN". In: 3rd International Conference on Advanced Computing and Communication Technologies (ACCT), pp. 236-240, April 2013.

[24] Liu Y, Xu K, Luo Z, Chen L. "A reliable clustering algorithm base on LEACH protocol in wireless mobile sensor networks". In: IEEE 2nd International Conference on Mechanical and Electrical Technology (ICMET), pp. 692-696, 2010.

[25] Shukla, Kajal, "Research On Energy Efficient Routing Protocol LEACH For Wireless Sensor Networks". International Journal of Engineering Research \& Technology (IJERT). Vol. 2, Issue 3, pp. 1-5, 2013.

\section{BIOGRAPHIES OF AUTHORS}

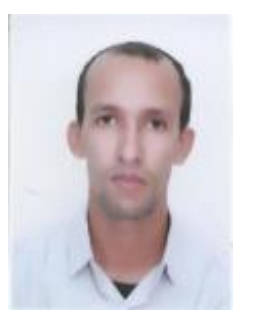

Abderrahmane El Aalaoui was born in Tantan Morocco. He's a member in the Physics department, Team Communication and Detection Systems, Faculty of Sciences, University of AbdelmalekEssaâdi, Tetouan Morocco, and his research area is wireless intelligent sensors and theirs applications, routing and real time protocols for energy optimization in wireless sensors networks. He obtained a Master's degree in Electrical and Computer Engineering from Faculty of Sciences and Technology of Marrakech Morocco.

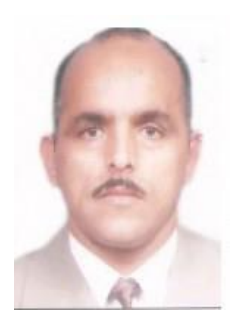

Abderrahmane Hajraoui is a professor of the Higher Education at University of AbdelmalekEssaâdi. He's a director thesis in the Physics department, Communication and Detection Systems laboratory, Faculty of sciences, AbdelmalekEssaâdi University, Tetouan, Morocco. His research areas are Signal and Image Processing, Automatics, Automation systems, Simulation systems, Antennas and radiation, Microwave devices and intelligent wireless sensors networks. 